Research Article

\title{
Optical Quality Assessment in Patients with Unilateral Congenital Ptosis: A Matched Case-Control Study
}

\author{
Jianqin Shen, Hongguang Cui, Xuyuan Tang, Miaomiao Zhu, and Wei Han (iD \\ Department of Ophthalmology, First Affiliated Hospital, College of Medicine, Zhejiang University, Hangzhou, China \\ Correspondence should be addressed to Wei Han; 1196013@zju.edu.cn
}

Received 14 April 2020; Accepted 30 October 2020; Published 17 November 2020

Academic Editor: Alejandro Cerviño

Copyright ( $\odot 2020$ Jianqin Shen et al. This is an open access article distributed under the Creative Commons Attribution License, which permits unrestricted use, distribution, and reproduction in any medium, provided the original work is properly cited.

\begin{abstract}
Background. To evaluate the differences in the corneal higher-order aberrations (HOAs) and optical quality of the ptosis eyes compared with the normal fellow eyes in the unilateral congenital ptosis patients. Methods. A matched case-control study was performed in 17 pairs of eyes in 17 unilateral congenital ptosis patients. The ptosis eye was enrolled in the ptosis group while the normal fellow eye was enrolled in the normal group. The HOAs obtained from Pentacam HR and the optical quality parameters obtained from Optical Quality Analysis System (OQAS) were compared between the two groups. Results. There were significant differences in a vertical coma and vertical trefoil on the anterior corneal surface between the ptosis group and the normal group $(p<0.05)$. The OQAS parameters in the ptosis group were significantly different from those in the normal group $(p<0.05)$. The vertical fissure height (VFH) showed a significant correlation with the total HOAs, $3^{\text {rd }}$ HOAs, and vertical coma on the anterior corneal surface $(p<0.05)$. Conclusions. Compared with the normal fellow eyes, the ptosis eyes showed significantly degraded optical quality. The eyelid position was important for the changes of corneal HOAs in the ptosis eyes. The reasons for the discrepancy of the OQAS parameters between the ptosis eyes and normal fellow eyes were multiple.
\end{abstract}

\section{Introduction}

Congenital ptosis is a rare eyelid disease characterized by lower positioning of the upper eyelid presenting at birth or within the first year of life. It may be either unilateral or bilateral. Although the congenital ptosis commonly presents benign nonprogressive progress, it might be closely associated with visual function impairment [1-3].

Subjective visual quality is currently the major and common clinical criterion to assess the preoperative visual function of ptosis and determine the surgical intervention opportunity. However, the results of subjective visual quality are more dependent on the subjective response of the patients and hence error-prone. In comparison, the results of objective visual quality are more accurate and concrete. Therefore, the preoperative evaluation of objective visual quality in the ptosis eye is critical and could be an important approach to the visual function assessment.

Previous studies have suggested that drooping upper eyelid pressure can cause changes in corneal curvature which played an important role in objective visual quality. Grey and Yap found that the ocular with-the-rule astigmatism was significantly increased when the lid aperture was deliberately narrowed [4]. Buehren's study showed that the changes in corneal topography and HOAs were directly related to the force exerted by the lowered upper lid while reading [5].

Unlike the mechanism of upper eyelid drooping when reading or squeezing the eye, the upper eyelid drooping and loosening in ptosis eyes are due to the loss of muscular or nerve function. Therefore, the influence of the upper eyelid pressure on cornea in the ptosis eye might be different. It might be mild but persistent compared with the conditions of squeezing or reading. To the best of our knowledge, there are little studies focusing on the objective visual quality changes in ptosis eyes. Further studies to elucidate this issue would be desired.

In this study, we investigated the differences in the corneal higher-order aberrations (HOAs) and Optical Quality Analysis System (OQAS) parameters between the ptosis eyes and the normal fellow eyes. Furthermore, the 
correlation between the severity of the ptosis and visual quality parameters was analyzed. To the best of our knowledge, this is the first study regarding the investigation of the anterior and posterior corneal HOAs as well as OQAS parameters in unilateral ptosis cases. Our work could help to further elucidate the causes of visual impairment in ptosis patients and develop a new robust approach to evaluating the visual function in ptosis.

\section{Subjects and Methods}

2.1. Subjects. Clinical records of 17 pairs of eyes in 17 unilateral ptosis patients were collected at our Medical Center from December 2017 to May 2019. The ptosis eyes (17 eyes) were enrolled in the ptosis group while the normal fellow eyes (17 eyes) were enrolled in the normal group. Patients with corneal surface-altering diseases such as pterygium, keratoconus, or contact lens wearing and with other ocular or orbital surgeries were excluded.

The study was approved by the Institutional Review Board. Written informed consent was obtained from legal parent/guardian for participants less than 18 years old and from participants directly for those 18 years or older. The tenets of the Declaration of Helsinki were followed for all study procedures.

\section{Procedure}

All subjects underwent routine clinical assessments, topographic measurements, and visual quality evaluations. All the measurements were completed by Jianqin Shen.

(1) Routine examinations involved uncorrected visual acuity (UCVA), best-corrected visual acuity (BCVA), intraocular pressure, slit-lamp examination, fundus examination, and cycloplegic refraction (cycloplegic refraction measurement was performed after the examination of Pentacam HR and OQAS). Before the refraction, $1 \%$ atropine sulphate ointment (QN for three days) was administered to the subjects less than 8 years, and $0.5 \%$ tropicamide eye drops (four times with five-minute intervals) was administered to the subjects more than 8 years. Autorefraction was performed using an autorefractor (RKF2, Canon, Japan)

(2) Vertical fissure height (VFH): VFHs were obtained from the external eye photographs captured by OCULUS Keratograph (Oculus, Wetzlar, Germany) (Figure 1).

(3) Corneal HOAs: corneal HOAs on the anterior surface and posterior surface were measured by Pentacam HR (Oculus, Wetzlar, Germany). The measurements were performed in a dark room. Patients were asked to blink twice to smooth the corneal surface in order to minimize the influence of tear film on corneal imaging. For uniform measurements, the upper eyelid was lifted in all eyes mechanically just before the camera rotated. The Pentacam HR then immediately started scanning the

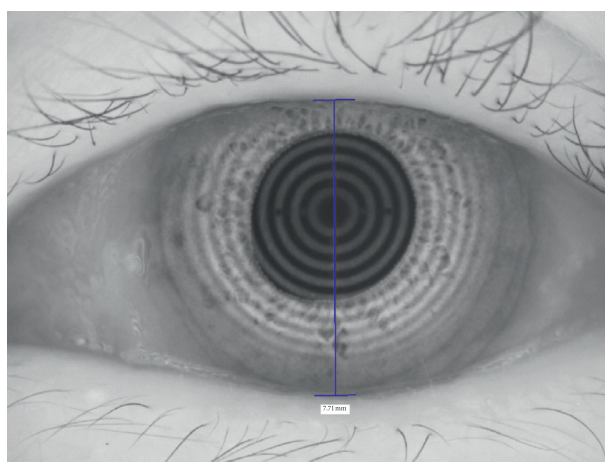

FIgURE 1: A diagrammatic description of the vertical fissure height measurement on the photograph captured by OCULUS Keratograph (the blue marker).

cornea. Measurements marked as "OK" quality were considered valid. Corneal HOAs of the anterior surface and posterior surface were analyzed over a $6.0 \mathrm{~mm}$ central diameter. Corneal HOA parameters include (1) the root mean square (RMS) of the total, $3^{\text {rd }}, 4^{\text {th }}$, and $5^{\text {th }}$ HOAs on the anterior and posterior corneal surfaces and (2) the Zernike coefficients from $3^{\text {rd }}$ up to $5^{\text {th }}$ orders on the anterior and posterior corneal surfaces.

3.1. OQAS Parameters. Visual quality evaluations were performed by OQAS (OQAS II; Visiometrics SL, Terrassa, Spain). The measurements were performed with a $4.0 \mathrm{~mm}$ central diameter, which was a standard size used in clinical double-pass studies. For all subjects, the actual pupil sizes as detected by the OQAS were always larger than $4 \mathrm{~mm}$. Care was taken to avoid the pressure of fingers on the eyeball when lifting the eyelid to expose the entire corneal zone. For uniform measurement, the refractive error of the eye was corrected by the spherical lens and the astigmatism was corrected by the cylindrical lens. All the measurements were conducted after blinking twice in order to minimize the influence of tear film on the light scattering.

The following parameters were measured to quantify the optical quality:

(1) Objective scatter index (OSI): the ratio of the peripherally annular area light versus that of the central peak in the acquired double-pass image, which quantifies the intraocular scatter [6]. The lower OSI value indicates a better optical quality.

(2) Modulation transfer function (MTF) cut-off: the cutoff point of the MTF curve on the $x$-axis, which is directly computed from the point spread function (PSF) [7]. The higher MTF cut-off value indicates a better optical quality.

(3) Strehl ratio (SR): the ratio of peak focal intensities in aberrated PSF and ideal PSF, which ranges between 0 and 1.0. The larger SR indicates a better optical quality $[7,8]$. 
(4) Optical quality analysis system values (OVs) (OV $100 \%$, OV $20 \%$, and OV $9 \%$ ): three different spatial frequencies of the MTF values, which describe optical quality for three contrast conditions that are commonly used in ophthalmology practice [9-11].

\section{Data Analysis and Statistics}

All data were expressed as the mean \pm standard deviation. Statistical analyses were performed using SPSS 22.0 software (SPSS, Chicago, IL, USA). The normality of all data samples was first checked using the Shapiro-Wilk test. When the parametric analysis was possible, paired $t$-tests for paired data were used for the comparisons. When the parametric analysis was not possible, Wilcoxon signed-rank tests for paired data were used. For the normal distribution, the correlation between variables was analyzed using the Pearson bivariate correlation. For abnormal distribution, the correlation between variables was analyzed using the Spearman bivariate correlation. The difference was considered statistically significant when the $p$ value is smaller than 0.05 .

\section{Results}

The study involved 17 ptosis patients with a mean age of $25.00 \pm 11.95$ years $(6-47$ years $)$.

5.1. BCVA. The mean and standard deviations of bestcorrected $\log$ MAR visual acuity were $0.115 \pm 0.120$ in the ptosis group and $0.017 \pm 0.038$ in the normal fellow group. There was a significant difference in BCVA between the two groups $(p<0.05)($ Table 1$)$.

5.2. Spherical Equivalent (SE) and Astigmatism. The mean and standard deviations of SE were $-2.07 \pm 3.61 \mathrm{D}$ in the ptosis group and $-1.96 \pm 3.03 \mathrm{D}$ in the normal fellow group. There was no significant difference in SE between the two groups $(p>0.05)$ (Table 1$)$.

The mean and standard deviations of astigmatism were $-0.94 \pm 0.95 \mathrm{D}$ in the ptosis group and $-0.68 \pm 0.80 \mathrm{D}$ in the normal fellow group. There was no significant difference in astigmatism between the two groups $(p>0.05)$ (Table 1$)$.

5.3. VFH. The mean and standard deviations of VFH were $6.26 \pm 1.00 \mathrm{~mm}$ in the ptosis group and $9.13 \pm 0.59 \mathrm{~mm}$ in the normal group. There was a significant difference in VFH between the two groups $(p<0.05)$ (Table 1).

5.4. Corneal HOAs. The anterior corneal HOAs and Zernike coefficients $\left(3^{\text {rd }}\right.$ to $5^{\text {th }}$ order) are shown in Table 2. There was no significant difference in total HOA RMS, $3^{\text {rd }}$ HOA RMS, $4^{\text {th }}$ HOA RMS, and $5^{\text {th }}$ HOA RMS on the anterior corneal surface between the ptosis and the normal eyes $(p>0.05)$ (Figure 2). There were significant differences in the vertical coma $\left(Z_{3}^{-1}\right)$ and vertical trefoil $\left(Z_{3}^{-3}\right)$ on the anterior corneal surface between the ptosis and the fellow eyes $(p<0.05)$
TABle 1: Clinical data analysis for the eyes of the ptosis group and the normal fellow group.

\begin{tabular}{lccc}
\hline & Ptosis eye & Normal fellow eye & $p$ value \\
\hline BCVA (LogMAR) & $0.115 \pm 0.120$ & $0.017 \pm 0.038$ & $0.007^{\mathrm{b} *}$ \\
SE (D) & $-2.07 \pm 3.61$ & $-1.96 \pm 3.03$ & $0.495^{\mathrm{b}}$ \\
Astigmatism (D) & $-0.94 \pm 0.95$ & $-0.68 \pm 0.80$ & $0.237^{\mathrm{b}}$ \\
VFH (mm) & $6.26 \pm 1.00$ & $9.13 \pm 0.59$ & $<0.001^{\mathrm{a} *}$ \\
\hline
\end{tabular}

SE: spherical equivalent; VFH: vertical fissure height; a: paired $t$-tests; $\mathrm{b}$ : Wilcoxon signed-rank tests; ${ }^{*}$ statistical significance $(p<0.05)$.

(Figure 3). The rest of the anterior corneal Zernike coefficients from the third to the fifth order showed no significant difference $(p>0.05)$.

The posterior corneal HOAs and Zernike coefficients (3rd to 5th order) are shown in Table 2. There was no significant difference in the total HOA RMS, 3rd HOA RMS, 4th HOA RMS, and $5^{\text {th }}$ HOA RMS of the posterior corneal surface between the ptosis and the normal eyes $(p>0.05)$ (Figure 2). There were significant differences in vertical trefoil $\left(Z_{3}^{-3}\right)$, oblique trefoil $\left(Z_{3}^{3}\right)$, vertical coma $\left(Z_{3}^{-1}\right)$, and secondary astigmatism $\left(Z_{4}^{2}\right)$ on the posterior corneal surface between the ptosis and the fellow eyes $(p<0.05)$ (Figure 3$)$. The rest of the posterior corneal Zernike coefficients from the third to the fifth order showed no significant difference $(p>0.05)$.

5.5. OQAS Parameters. Table 3 shows OQAS parameter comparisons between the ptosis group and the normal group. The ptosis group demonstrated statistically higher OSI and lower MTF, SR, OV100\%, OV20\%, and OV9\% than the normal fellow eye group $p<0.05$ (Figure 4).

5.6. The Correlation between BCVA and VFH. There was no significant correlation between VFH and BCVA (Spearman correlation coefficient $r=-0.053, p=0.840$ ).

5.7. The Correlation between SE and VFH. There was no significant correlation between SE and VFH (Spearman correlation coefficient $r=-0.070, p=0.790$ ).

\section{The Correlation between Corneal HOAs and VFH}

Table 4 shows that the VFH was significantly correlated with the anterior corneal total HOA RMS, $3^{\text {rd }}$ RMS, and vertical coma $\left(Z_{3}^{-1}, Z_{3}^{-1} \mathrm{RMS}\right)(p<0.05)$ (Figure 5$)$. The rest of the corneal HOAs showed no significant correlation with VFH $(p>0.05)$.

6.1. The Correlation between the OQAS Parameters and VFH. Table 5 shows that there was no significant correlation between the VFHs and the OQAS parameters $(p>0.05)$.

\section{Discussion}

Objective visual quality is an important criterion for visual function evaluation. The objective visual quality is mainly affected by aberrations, intraocular scatter, and 
TABLE 2: Comparison of corneal higher-order aberrations (HOAs) between the ptosis group and the normal group.

\begin{tabular}{|c|c|c|c|c|c|c|}
\hline & \multicolumn{3}{|c|}{ Anterior corneal HOAs } & \multicolumn{3}{|c|}{ Posterior corneal HOAs } \\
\hline & Ptosis & Normal & $p$ & Ptosis & Normal & $p$ \\
\hline Total HOA RMS & $0.565 \pm 0.149$ & $0.507 \pm 0.171$ & $0.119^{\mathrm{a}}$ & $0.230 \pm 0.052$ & $0.223 \pm 0.066$ & $0.723^{\mathrm{b}}$ \\
\hline $3^{\text {rd }}$ HOA RMS & $0.436 \pm 0.184$ & $0.369 \pm 0.196$ & $0.074^{\mathrm{a}}$ & $0.154 \pm 0.063$ & $0.130 \pm 0.066$ & $0.298^{\mathrm{b}}$ \\
\hline $4^{\text {th }}$ HOA RMS & $0.297 \pm 0.081$ & $0.298 \pm 0.104$ & $0.970^{\mathrm{a}}$ & $0.152 \pm 0.030$ & $0.166 \pm 0.040$ & $0.055^{\mathrm{a}}$ \\
\hline $5^{\text {th }}$ HOA RMS & $0.132 \pm 0.068$ & $0.102 \pm 0.048$ & $0.122^{\mathrm{a}}$ & $0.057 \pm 0.024$ & $0.056 \pm 0.029$ & $0.943^{\mathrm{b}}$ \\
\hline$Z_{3}^{-3}$ & $0.105 \pm 0.215$ & $-0.062 \pm 0.213$ & $0.032^{\mathrm{a} *}$ & $-0.097 \pm 0.074$ & $-0.009 \pm 0.108$ & $0.013^{\mathrm{a} *}$ \\
\hline$Z_{3}^{-1}$ & $-0.184 \pm 0.299$ & $0.006 \pm 0.286$ & $0.017^{\mathrm{a} *}$ & $0.056 \pm 0.061$ & $-0.008 \pm 0.059$ & $0.004^{\mathrm{a} *}$ \\
\hline$Z_{3}^{1}$ & $-0.015 \pm 0.195$ & $-0.015 \pm 0.196$ & $0.996^{\mathrm{a}}$ & $0.017 \pm 0.032$ & $-0.011 \pm 0.027$ & $0.051^{\mathrm{a}}$ \\
\hline$Z_{3}^{3}$ & $-0.013 \pm 0.117$ & $0.009 \pm 0.119$ & $0.663^{\mathrm{a}}$ & $0.023 \pm 0.068$ & $-0.038 \pm 0.067$ & $0.023^{\mathrm{a} *}$ \\
\hline$Z_{4}^{-4}$ & $0.012 \pm 0.067$ & $0.017 \pm 0.111$ & $0.888^{\mathrm{a}}$ & $-0.001 \pm 0.041$ & $-0.009 \pm 0.040$ & $0.636^{\mathrm{b}}$ \\
\hline$Z_{4}^{-2}$ & $-0.000 \pm 0.046$ & $0.018 \pm 0.050$ & $0.352^{\mathrm{a}}$ & $-0.003 \pm 0.017$ & $-0.000 \pm 0.014$ & $0.631^{\mathrm{a}}$ \\
\hline$Z_{4}^{0}$ & $0.198 \pm 0.129$ & $0.230 \pm 0.089$ & $0.216^{\mathrm{a}}$ & $-0.141 \pm 0.041$ & $-0.151 \pm 0.034$ & $0.084^{\mathrm{b}}$ \\
\hline$Z_{4}^{4}$ & $0.011 \pm 0.139$ & $-0.006 \pm 0.065$ & $0.519^{\mathrm{a}}$ & $-0.023 \pm 0.028$ & $-0.009 \pm 0.031$ & $0.017^{\mathrm{a} *}$ \\
\hline$Z_{4}^{4}$ & $-0.063 \pm 0.107$ & $-0.044 \pm 0.140$ & $0.620^{\mathrm{a}}$ & $-0.030 \pm 0.031$ & $-0.040 \pm 0.027$ & $0.322^{\mathrm{a}}$ \\
\hline$Z_{5}^{-5}$ & $0.009 \pm 0.077$ & $-0.007 \pm 0.072$ & $0.528^{\mathrm{a}}$ & $-0.023 \pm 0.025$ & $-0.016 \pm 0.0340$ & $0.368^{\mathrm{b}}$ \\
\hline$Z_{5}^{-3}$ & $-0.006 \pm 0.078$ & $0.011 \pm 0.041$ & $0.868^{\mathrm{b}}$ & $0.005 \pm 0.026$ & $-0.005 \pm 0.022$ & $0.260^{\mathrm{a}}$ \\
\hline$Z_{5}^{-1}$ & $0.009 \pm 0.079$ & $-0.005 \pm 0.053$ & $0.460^{\mathrm{a}}$ & $0.005 \pm 0.029$ & $0.0080 \pm 0.027$ & $0.650^{\mathrm{a}}$ \\
\hline$Z_{5}^{1}$ & $0.008 \pm 0.021$ & $0.008 \pm 0.020$ & $0.946^{\mathrm{a}}$ & $0.002 \pm 0.013$ & $-0.006 \pm 0.011$ & $0.113^{\mathrm{b}}$ \\
\hline$Z_{5}^{3}$ & $-0.014 \pm 0.027$ & $-0.009 \pm 0.025$ & $0.618^{\mathrm{a}}$ & $-0.005 \pm 0.016$ & $0.005 \pm 0.015$ & $0.142^{\mathrm{a}}$ \\
\hline$Z_{5}^{5}$ & $0.021 \pm 0.053$ & $-0.009 \pm 0.047$ & $0.097^{\mathrm{a}}$ & $-0.002 \pm 0.030$ & $-0.007 \pm 0.031$ & $0.672^{\mathrm{a}}$ \\
\hline
\end{tabular}

a: paired $t$-tests; b: Wilcoxon signed-rank tests; ${ }^{*}$ statistical significance $(p<0.05)$; RMS: root mean square; HOAs: higher-order aberrations.

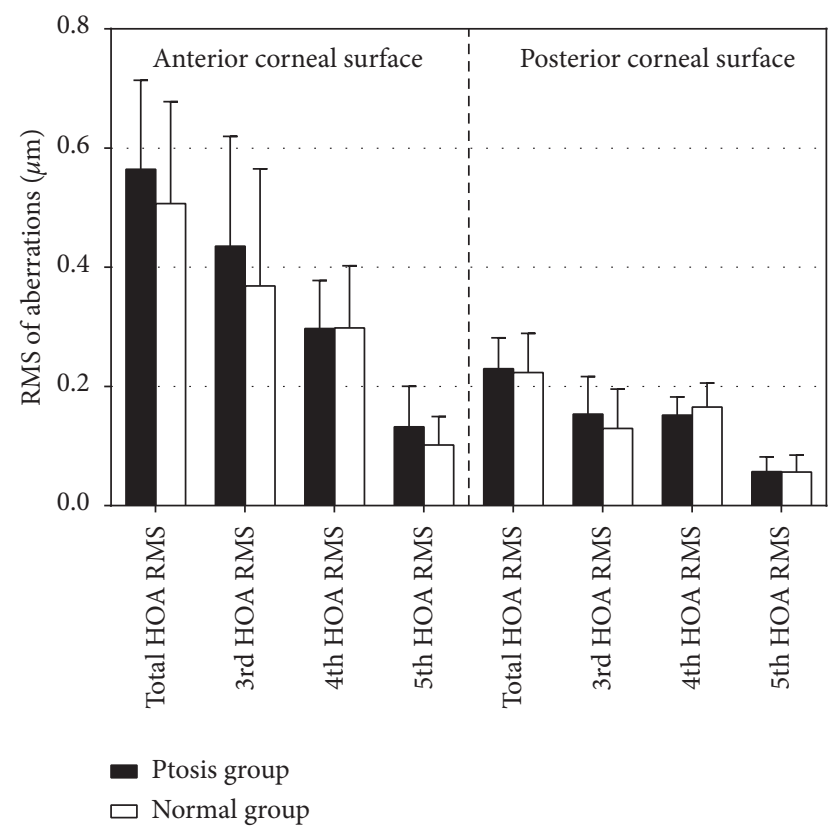

FIGURE 2: Comparisons of the total and third-order to five-order higher-order aberrations (RMS HOAs) of the anterior and posterior corneal surface between the ptosis group and the normal fellow group. ${ }^{*} p<0.05$.

diffraction [12]. Aberration in ocular optical disorders has been well documented in previous studies. Eyelid position has been found to be an important factor contributing to the aberration changes $[4,5,13,14]$. For objective visual quality changes in ptosis, nearly all studies have focused on the differences in lower-order aberration between ptosis eyes and normal eyes [15-18], and only a few studies have aimed at the HOAs changes in ptosis eyes. However, there are still no studies investigating the corneal HOAs and intraocular scatter changes in ptosis eyes.

Currently, there are mainly two grading systems of ptosis levels [19, 20]: (1) based on the VFH and (2) based on the function of the levator muscle. Since the present work focused on the influence of eyelid position in the ocular optical quality, we followed the definition based on the VFH. According to the VFH data, our subjects can be categorized as the mild-moderate degree ptosis.

Amblyopia and refractive errors have aroused much attention in ptosis over the past decades. Amblyopia is one of the most common reasons for visual impairment in congenital ptosis, and the rate of amblyopia in congenital ptosis has been reported to be higher than the one in normal eyes [21]. Eyelid occlusion and significant refractive error were two leading causes of amblyopia in ptosis. Our results showed there was a significant difference in BCVA between the two groups, but there was no significant difference in spherical equivalent refraction. It can be speculated that the eyelid occlusion was the main cause of amblyopia in ptosis eyes. The association between BCVA and VFH was relatively weak and not statistically significant. This was probably because the degrees of most ptosis eyes in our study are moderate with similar VFH values. As a result, the discrepancy of the pupil zone blocked by the ptosis eyes lid was too little to cause a significantly different effect on amblyopia.

The pooled prevalence of myopia was much higher in the ptosis population than the normal population [22]. The mechanisms of myopia in ptosis eyes are multifactorial and complicated. Form deprivation by the eyelid, the defocused image on retinal, and the eyelid pressure on the eyeball might be the main causes [23-25]. Our results showed that there was no significant difference in refractive errors 


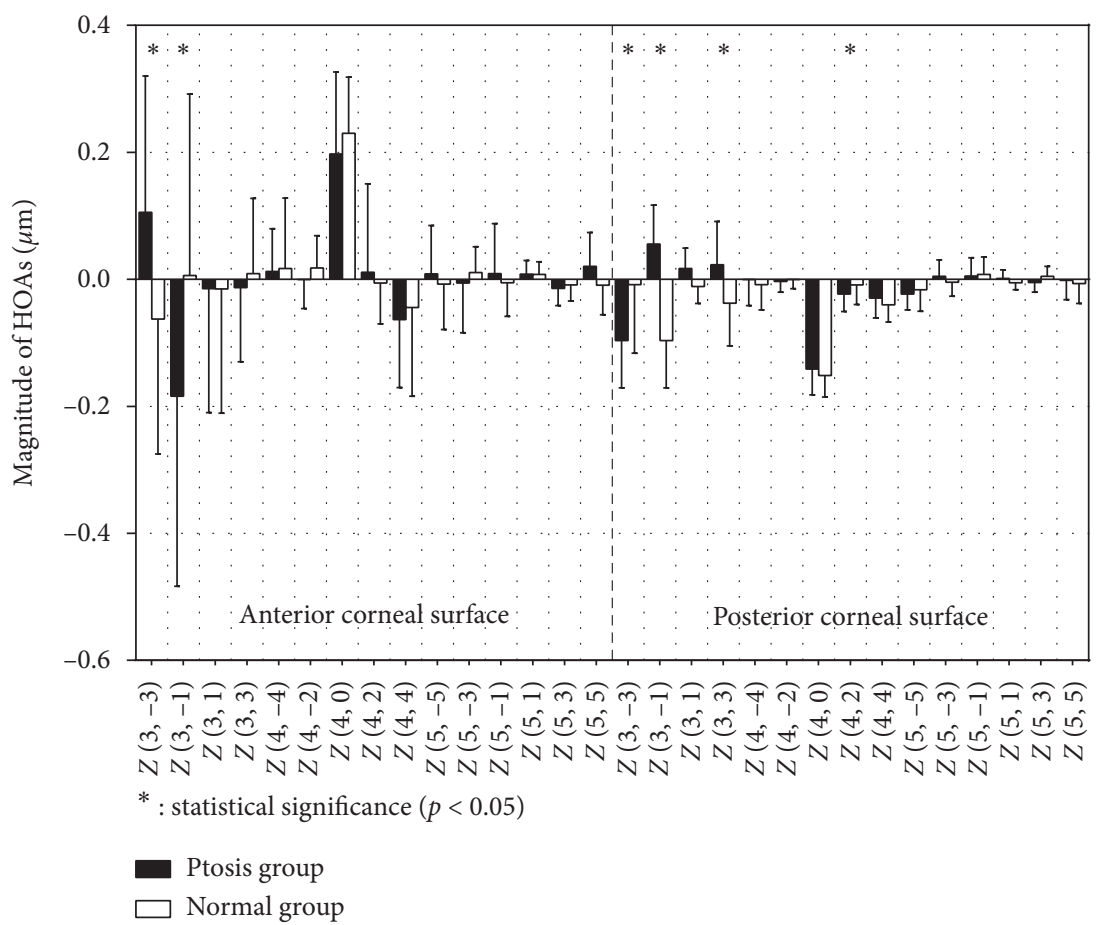

Figure 3: Comparisons of the Zernike coefficients (3rd to 5th order) of the anterior and posterior corneal surface between the ptosis group and the normal fellow group.

TABLE 3: Comparison of the OQAS parameters between the ptosis group and the normal group.

\begin{tabular}{lccc}
\hline & Ptosis group & Normal group & $p$ Value \\
\hline OSI & $2.32 \pm 2.36$ & $0.61 \pm 0.45$ & $0.003^{\mathrm{b} *}$ \\
MTF & $23.07 \pm 14.09$ & $41.09 \pm 10.13$ & $0.001^{\mathrm{a} *}$ \\
SR & $0.14 \pm 0.07$ & $0.21 \pm 0.08$ & $0.019^{\mathrm{a} *}$ \\
OV100\% & $0.77 \pm 0.49$ & $1.37 \pm 0.36$ & $<0.001^{\mathrm{a} *}$ \\
OV20\% & $0.55 \pm 0.34$ & $0.99 \pm 0.30$ & $0.003^{\mathrm{b} *}$ \\
OV9\% & $0.34 \pm 0.19$ & $0.60 \pm 0.19$ & $0.001^{\mathrm{a} *}$ \\
\hline
\end{tabular}

a: paired $t$-tests; b: Wilcoxon signed-rank tests; ${ }^{*}$ statistical significance $p<0.05$; MTF: modulation transfer function; SR: Strehl ratio; OSI: objective scatter index; OV: Optical Quality Analysis System Value; OQAS: Optical Quality Analysis System.

between the ptosis and the normal group, which was not in line with the literature. The correlation between the refractive errors and the VFH was not statistically significant. This could also be ascribed to the little discrepancy of VFH in our patients.

The anterior corneal surface is a major contributor to the total HOAs of the eye. The effect on the corneal shape of the eyelid mainly is exerted on the anterior corneal surface. Thus, we focused on the corneal HOAs in this study. In the present study, both the vertical coma and the vertical trefoil of the anterior corneal HOAs showed a significant difference between the ptosis group and the normal group. Similar changes were found in other palpebral fissure narrowed conditions. Buehren's study showed corneal vertical coma and trefoil changed in both magnitude and direction after reading [5]. The upper eyelid pressure exerted on the cornea in a vertical direction is the likely optical explanation for the significant increase in vertical corneal aberrations when the upper lid drops. Our findings are partly in line with the observations of the total ocular aberrations in ptosis and other palpebral fissure narrowed conditions. Kumar et al. reported that the vertical trefoil of the total ocular showed a significant difference between the ptosis and the normal eyes in Indian children, but the vertical coma showed no significant difference between the two groups [26]. We speculated that it was probably due to the different anatomical characteristics of eyelid between different races. Asian eyes have smaller vertical palpebral apertures and thicker upper eyelid and skin, all of which may contribute to the increased optical effects of the upper eyelid forces on cornea [27]. Changes in the ocular vertical coma while deliberately narrowing the palpebral fissure have been found to be significant in our previous research [14], but the significance level is much stronger in the present study. The reasons for the differences are multiple. Unlike the condition of deliberately squeezing eyelids, ptosis cases do not have an additional force from the contraction of muscle on the eyeball. In addition, the lid pressure distribution in congenital ptosis is uniform while the pressure distribution in deliberately squeezing state might be more localized. Moreover, congenital patients may develop head positions which could cancel the excess upper eyelid pressure to some degree [26].

Furthermore, we found that the VFH had a significantly negative correlation with the total HOAs RMS and the $3^{\text {rd }}$ HOA RMS on the anterior corneal surface. The third-order HOAs are the dominant aberrations for most eyes in general $[28,29]$. Therefore, our results indicated that the eyes with 


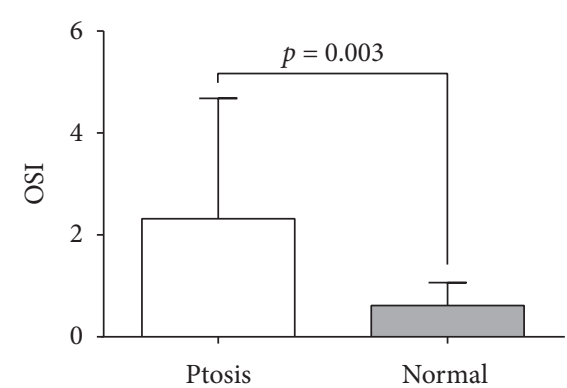

(a)

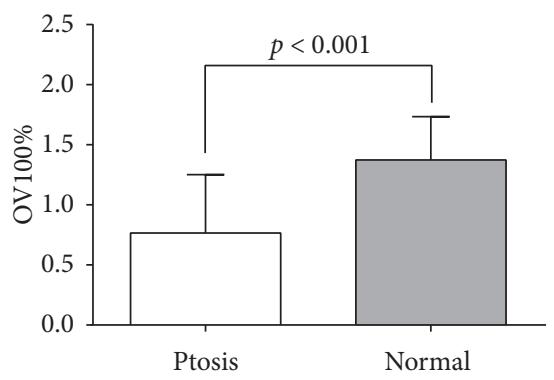

(d)

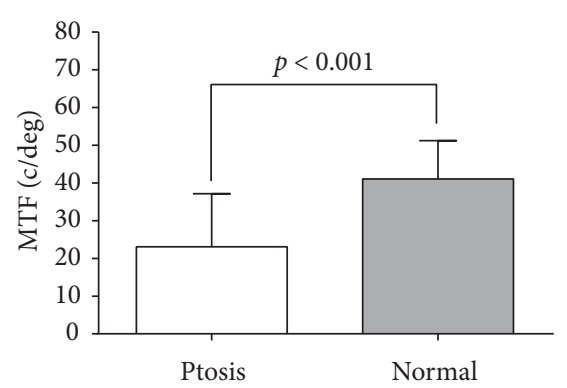

(b)

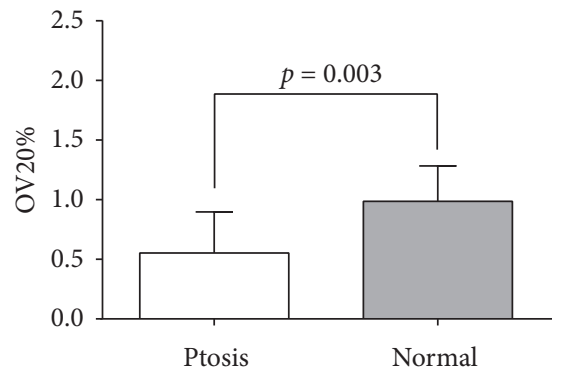

(e)

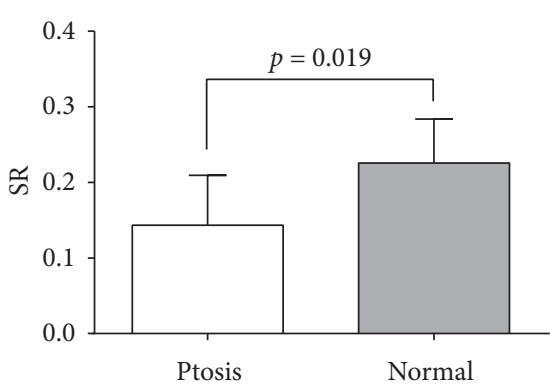

(c)

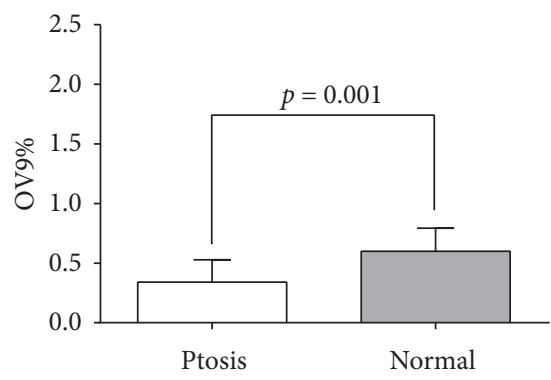

(f)

FIgURE 4: Comparisons of the OQAS parameter between the ptosis group and the normal group.

TABLE 4: The correlation between the corneal HOAs and VFH.

\begin{tabular}{lcccc}
\hline & \multicolumn{2}{c}{$\begin{array}{c}\text { Anterior corneal } \\
\text { surface }\end{array}$} & $\begin{array}{c}\text { Posterior corneal } \\
\text { surface }\end{array}$ \\
& Coefficient $\mathrm{r}$ & $p$ value & Coefficient $\mathrm{r}$ & $p$ value \\
\hline Total HOA RMS & -0.552 & $0.022^{\mathrm{c} *}$ & 0.276 & $0.283^{\mathrm{c}}$ \\
$3^{\text {rd }}$ HOA RMS & -0.567 & $0.018^{\mathrm{c} *}$ & 0.288 & $0.262^{\mathrm{c}}$ \\
$4^{\text {th }}$ HOA RMS & 0.202 & $0.437^{\mathrm{c}}$ & -0.016 & $0.950^{\mathrm{c}}$ \\
$Z_{3}^{-3}$ & -0.478 & $0.052^{\mathrm{c}}$ & -0.329 & $0.197^{\mathrm{c}}$ \\
$Z_{3}^{-1}$ & 0.726 & $0.001^{\mathrm{c} *}$ & -0.295 & $0.251^{\mathrm{c}}$ \\
$Z_{3}^{1}$ & -0.073 & $0.780^{\mathrm{c}}$ & -0.073 & $0.781^{\mathrm{c}}$ \\
$Z_{3}^{3}$ & -0.037 & $0.887^{\mathrm{c}}$ & -0.072 & $0.784^{\mathrm{c}}$ \\
$Z_{4}^{-4}$ & -0.145 & $0.578^{\mathrm{c}}$ & 0.157 & $0.546^{\mathrm{c}}$ \\
$Z_{4}^{-2}$ & 0.437 & $0.080^{\mathrm{c}}$ & 0.098 & $0.708^{\mathrm{c}}$ \\
$Z_{4}^{0}$ & 0.318 & $0.213^{\mathrm{c}}$ & -0.112 & $0.669^{\mathrm{c}}$ \\
$Z_{4}^{2}$ & -0.330 & $0.195^{\mathrm{c}}$ & 0.036 & $0.892^{\mathrm{c}}$ \\
$Z_{4}^{4}$ & 0.261 & $0.312^{\mathrm{c}}$ & 0.137 & $0.600^{\mathrm{c}}$ \\
$Z_{3}^{-3}$ (RMS) & -0.254 & $0.326^{\mathrm{c}}$ & 0.328 & $0.198^{\mathrm{c}}$ \\
$Z_{3}^{-1}$ (RMS) & -0.674 & $0.003^{\mathrm{c} *}$ & -0.134 & $0.609^{\mathrm{c}}$ \\
$Z_{3}^{1}$ (RMS) & -0.163 & $0.531^{\mathrm{d}}$ & -0.287 & $0.264^{\mathrm{d}}$ \\
$Z_{3}^{3}$ (RMS) & -0.254 & $0.326^{\mathrm{c}}$ & 0.235 & $0.365^{\mathrm{c}}$ \\
$Z_{4}^{-4}$ (RMS) & 0.018 & $0.946^{\mathrm{c}}$ & -0.208 & $0.423^{\mathrm{d}}$ \\
$Z_{4}^{-2}$ (RMS) & -0.037 & $0.888^{\mathrm{d}}$ & -0.144 & $0.581^{\mathrm{c}}$ \\
$Z_{4}^{0}$ (RMS) & 0.361 & $0.155^{\mathrm{c}}$ & 0.112 & $0.669^{\mathrm{c}}$ \\
$Z_{4}^{2}$ (RMS) & -0.247 & $0.340^{\mathrm{c}}$ & 0.117 & $0.653^{\mathrm{c}}$ \\
$Z_{4}^{4}$ (RMS) & -0.067 & $0.799^{\mathrm{c}}$ & 0.085 & $0.747^{\mathrm{d}}$ \\
\hline $\mathrm{c}$ & Rers & & &
\end{tabular}

c: Pearson's correlation; d: Spearman's correlation; ${ }^{*}$ statistical significance $p<0.05$, VFH: vertical fissure height.

severe ptosis may have a poorer optical quality induced by corneal HOAs. Moreover, there was a strong significant correlation $(p<0.01)$ between VFH and vertical coma (both magnitude and direction), which indicated that the eyelid position was the major factor impacting the corneal HOAs, particularly the vertical coma in ptosis eyes. The total HOAs, the 3rd HOA, and the vertical coma might be a potential robust optical criterion to assess the visual function and the severity of the ptosis eyes.

It was also noticeable that the significant changes of HOAs in the posterior corneal surface were observed in our ptosis group. The HOAs on the posterior corneal surface have been rarely reported in either ptosis eyes or other narrowed palpebral fissure conditions. Vertical coma, vertical trefoil, horizontal trefoil, and vertical secondary astigmatism were significantly different between the two groups. The differences were basically in accordance with the anterior corneal HOAs, which indicated that the upper eyelid pressure might affect not only the HOAs on the anterior surface but also the posterior surface. However, the exact and specific mechanisms were worthy of intensive investigation. We speculated that long-term and persistent pressure from the drooping eyelid might not only cause the instant distortion but also "remodel" the whole corneal contour, which involved the changes of the posterior corneal surface. Therefore, HOAs on the posterior corneal surface should be an important factor to affect the ocular optical performance in ptosis.

The OQAS can evaluate the full information on aberration, diffraction, and scattering $[8,30]$. OQAS has been successfully used to evaluate ocular optical quality in various eye diseases such as cataracts [31], pseudophakic eyes [11], dry eyes [32, 33], corneal surface disorders [34], even macular disorders [35], and exhibited good repeatability. However, still, no study was reported using the OQAS to assess the optical quality in ptosis eyes.

Our results showed significantly higher OSI and lower MTF, SR, OV100\%, OV20\%, and OV9\% in the ptosis group, which indicated a comprehensive compromised optical 


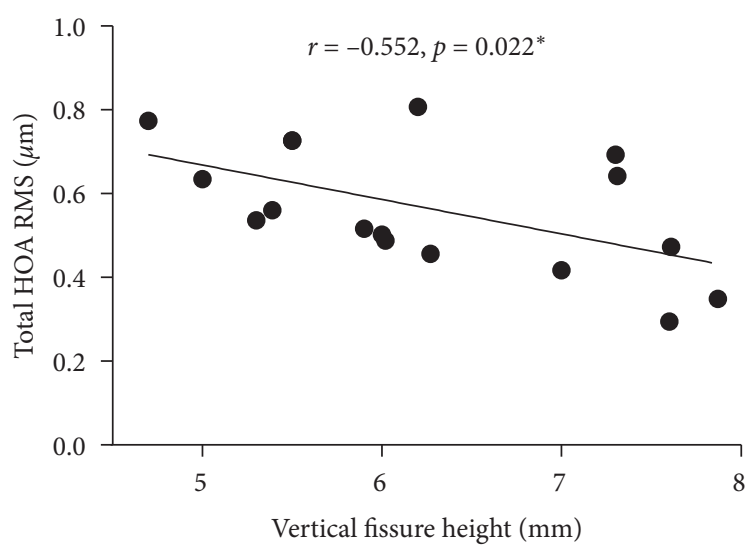

(a)

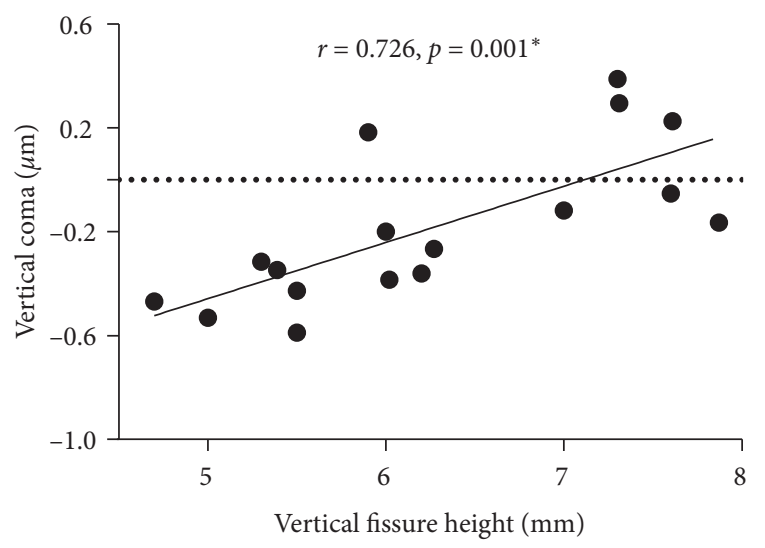

(c)

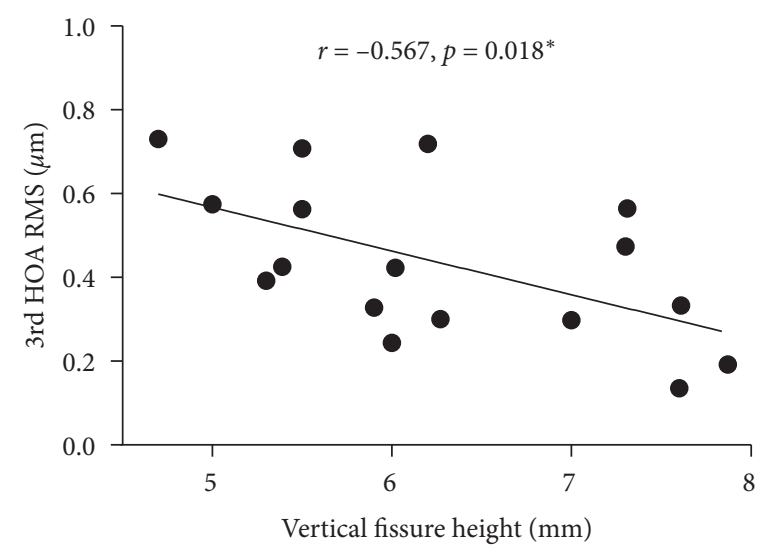

(b)

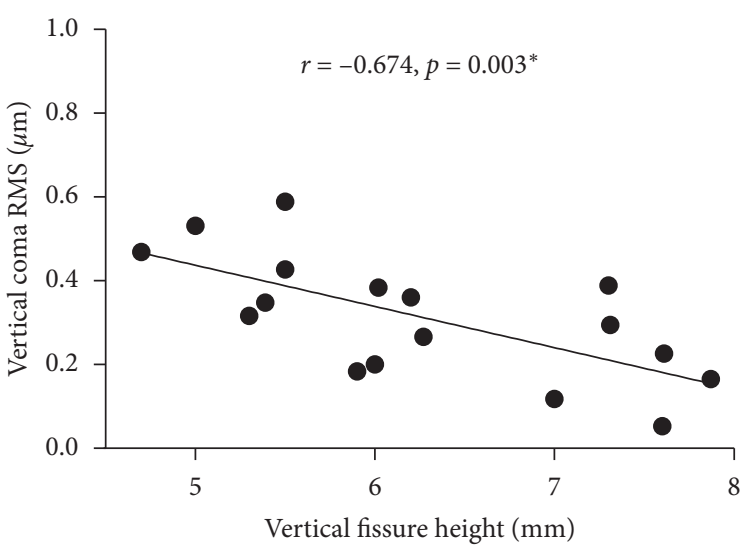

(d)

FIgURE 5: The correlations between VFH and total HOA RMS, 3rd RMS, and vertical coma of the anterior cornea surface. $* p<0.05$.

TABLE 5: The correlation between the OQAS parameters and VFH.

\begin{tabular}{lcc}
\hline & & VFH \\
& Coefficient $r$ & $p$ value \\
\hline OSI & 0.134 & $0.633^{\mathrm{d}}$ \\
MTF & -0.097 & $0.730^{\mathrm{c}}$ \\
SR & -0.172 & $0.539^{\mathrm{c}}$ \\
OV100\% & -0.087 & $0.757^{\mathrm{c}}$ \\
OV20\% & -0.109 & $0.698^{\mathrm{c}}$ \\
OV9\% & -0.026 & $0.925^{\mathrm{c}}$ \\
\hline
\end{tabular}

c: Pearson's correlation; d: Spearman's correlation; ${ }^{*}$ statistical significance $(p<0.05)$; VFH: vertical fissure height; MTF: modulation transfer function; SR: Strehl ratio; OSI : objective scatter index; OV: Optical Quality Analysis System Value.

quality for the ptosis eyes. Combined with the HOAs results, both the HOAs and intraocular scatter contributed to the degradation of the optical performances in ptosis eyes. There was no correlation between the VFH and neither of the OQAS parameters, which indicated that the reasons for poor optical quality in ptosis eyes might be multiple. A similar degree of $\mathrm{VFH}$ in our cases might be the major cause again here, as described above in the HOAs analysis. Nevertheless, other factors such as tear film and displacement of epithelial tissue might also be contributed to the reduction of objective visual quality in ptosis eye [36, 37].
No severe type of ptosis was involved in this work. Still, no studies regarding the relation between the VFH and HOAs or OQAS were reported, except the dioptre. However, our subjects showed a significant correlation between the VFH and the corneal HOAs. Based on the correlation trend in our data, it could be speculated that the severe type of ptosis might bring about more changes in the corneal HOAs. It should be emphasized that the pupil area was intact and round measurement of OQAS or HOAs, as the upper eyelid was lifted to ensure the exposure of the entire corneal zone. Therefore, the VFH should not affect the pupil size as well as the data acquirement of OQAS through the pupil zone.

There were limitations to our study. The sample size was small and hence there was a possible bias in statistical conclusion. In addition, the VFH was similar for all the ptosis eyes. Tear film was also a factor affecting the HOAs in ptosis eyes but was difficult to be measured for the ptosis eyes in the present study. Future studies regarding the tear film influence in the visual quality in ptosis eyes might be desired.

\section{Conclusions}

In conclusion, we found that the objective optical quality in the ptosis group was worse than that in the normal fellow group. Eyelid position is the critically important factor for the changes of HOAs on both the anterior and posterior 
corneal surfaces, particularly the vertical coma, in ptosis eyes. The reasons for the changes in the OQAS intraocular scatter parameters were complex. Further investigations with a larger sample size and involving more ptosis disease features, including eyelid thickness and more VFH degree, would help to unveil the mechanism underlining the etiology of visual impairment and develop the new approach to assessing visual function in the ptosis eyes.

\section{Data Availability}

The data that support the findings of this study are available from the corresponding author (Wei Han) upon reasonable request.

\section{Conflicts of Interest}

The authors declare that they have no conflicts of interest.

\section{Acknowledgments}

The work was supported by the Medical Health and Technology Project of Zhejiang Province (No. 2017198589) and the Zhejiang Provincial Natural Science Foundation of China (No. LQ18H120001).

\section{References}

[1] M. Marenco, I. Macchi, I. Macchi, E. Galassi, G. Massaro Giordano, and A. Lambiase, "Clinical presentation and management of congenital ptosis," Clinical Ophthalmology, vol. 11, no. 11, pp. 453-463, 2017.

[2] A. R. De Figueiredo, "Blepharoptosis," Semin Ophthalmology, vol. 25 , no. 3, pp. 39-51, 2010.

[3] J. R. SooHoo, B. W. Davies, F. D. Allard, and V. D. Durairaj, "Congenital ptosis," Survey of Ophthalmology, vol. 59, no. 5, pp. 483-492, 2014.

[4] C. Grey and M. Yap, "Influence of lid position on astigmatism," Optometry and Vision Science, vol. 63, no. 12, pp. 966-969, 1986.

[5] T. Buehren, M. J. Collins, and L. Carney, "Corneal aberrations and reading," Optometry and Vision Science, vol. 80, no. 2, pp. 159-166, 2003.

[6] M. Vilaseca, A. Padilla, J. C. Ondategui, M. Arjona, J. L. Güell, and J. Pujol, "Effect of laser in situ keratomileusis on vision analyzed using preoperative optical quality. Effect of laser in situ keratomileusis on vision analyzed using preoperative optical quality," Journal of Cataract and Refractive Surgery, vol. 36, no. 11, pp. 1945-1953, 2010.

[7] A. Saad, M. Saab, and D. Gatinel, "Repeatability of measurements with a double-pass system," Journal of Cataract \& Refractive Surgery, vol. 36, no. 1, pp. 28-33, 2010.

[8] J. A. Martínez-Roda, M. Vilaseca, J. C. Ondategui et al., "Optical quality and intraocular scattering in a healthy young population," Clinical and Experimental Optometry, vol. 94, no. 2, pp. 223-229, 2011.

[9] X. Liao, J. Lin, J. Tian, B. Wen, Q. Tan, and C. Lan, "Evaluation of optical quality: ocular scattering and aberrations in eyes implanted with diffractive multifocal or monofocal intraocular lenses," Current Eye Research, vol. 43, no. 6, pp. 696-701, 2018.

[10] X. Liao, J. Lin, J. Tian, B. Wen, Q. Tan, and C. Lan, "Clinical evaluation of optical quality and intraocular scattering after posterior chamber phakic intraocular lens implantation," Investigative Ophthalmology \& Visual Science, vol. 53, no. 6, pp. 3161-3166, 2012.

[11] X. W. Xiao, J. Hao, H. Zhang, and F. Tian, "Optical quality of toric intraocular lens implantation in cataract surgery," International Journal of Ophthalmology, vol. 8, no. 1, pp. 66-71, 2015.

[12] D. P. Piñero, D. Ortiz, and J. L. Alio, "Ocular scattering," Optometry and Vision Science, vol. 87, no. 9, pp. E682-E696, 2010.

[13] T. Buehren, M. J. Collins, and L. G. Carney, "Near work induced wavefront aberrations in myopia," Vision Research, vol. 45, no. 10, pp. 1297-1312, 2005.

[14] W. Han, W. Kwan, J. Wang, S. P. Yip, and M. Yap, "Influence of eyelid position on wavefront aberrations," Ophthalmic and Physiological Optics, vol. 27, no. 1, pp. 66-75, 2007.

[15] J. S. Paik, S. A. Kim, S. H. Park, and S. W. Yang, "Refractive error characteristics in patients with congenital blepharoptosis before and after ptosis repair surgery," Bio Med Central Ophthalmology, vol. 16, no. 1, p. 177, 2016.

[16] I. B. Simsek, B. Yilmaz, S. Yildiz, and O. Artunay, "Effect of upper eyelid blepharoplasty on vision and corneal tomographic changes measured by Pentacam," Orbit, vol. 34, no. 5, pp. 263-267, 2015.

[17] Y. K. Kim, J. H. In, and S. Y. Jang, "Changes in corneal curvature after upper eyelid surgery measured by corneal topography," Journal of Craniofacial Surgery, vol. 27, no. 3, pp. e235-e238, 2016.

[18] M. S. Zinkernagel, A. Ebneter, and D. Ammann-Rauch, "Effect of upper eyelid surgery on corneal topography," Archives of Ophthalmology, vol. 125, no. 12, pp. 1610-1612, 2007.

[19] J. Finsterer, "Ptosis: causes, presentation, and management," Aesthetic Plastic Surgery, vol. 27, no. 3, pp. 193-204, 2003.

[20] R. L. Anderson and R. S. Dixon, "Aponeurotic ptosis surgery," Archives of Ophthalmology, vol. 97, no. 6, pp. 1123-1128, 1979.

[21] G. J. Griepentrog, N. Diehl, and B. G. Mohney, "Amblyopia in childhood eyelid ptosis," American Journal of Ophthalmology, vol. 155, no. 6, pp. 1125-1128, 2013.

[22] A. Berry-Brincat and H. Willshaw, "Paediatric blepharoptosis: a 10-year review," Eye, vol. 23, no. 7, pp. 1554-1559, 2009.

[23] L. Huo, D. Cui, X. Yang et al., "A retrospective study: formdeprivation myopia in unilateral congenital ptosis," Clinical and Experimental Optometry, vol. 95, no. 4, pp. 404-409, 2012.

[24] A. Kasaee, A. Yazdani-Abyaneh, S. Z. Tabatabaie et al., "Assessing amblyogenic factors in 100 patients with congenital ptosis," International Journal of Ophthalmology, vol. 3, no. 4, pp. 328-330, 2010.

[25] S. D. Byard, V. Sood, and C. A. Jones, "Long-term refractive changes in children following ptosis surgery: a case series and a review of the literature," International Ophthalmology, vol. 34, no. 6, pp. 1303-1307, 2014.

[26] D. Ashok Kumar, Ms A. Agarwal, G. Prakash, N. Vajpayee Boptm, S. Packiyalakshmi, and A. Agarwal, "Effect of unilateral congenital ptosis on ocular higher order aberrations in children," Medical Hypothesis, Discovery \& Innovation in Ophthalmology, vol. 2, no. 3, pp. 86-91, 2013.

[27] M. T. Doxanas and R. L. Anderson, "Oriental eyelids," Archives of Ophthalmology, vol. 102, no. 8, pp. 1232-1235, 1984.

[28] H. C. Howland and B. Howland, "A subjective method for the measurement of monochromatic aberrations of the eye," Journal of the Optical Society of America, vol. 67, no. 11, pp. 1508-1518, 1977. 
[29] J. Liang and D. R. Williams, "Aberrations and retinal image quality of the normal human eye," Journal of the Optical Society of America, vol. 14, no. 11, pp. 2873-2883, 1997.

[30] F. Díaz-Doutón, A. Benito, J. Pujol, M. Arjona, J. L. Güell, and P. Artal, "Comparison of the retinal image quality with a Hartmann-shack wavefront sensor and a double-pass instrument," Optometry and Vision Science, vol. 47, no. 4, pp. 1710-1765, 2006.

[31] F. Cabot, A. Saad, C. McAlinden, N. M. Haddad, A. GriseDulac, and D. Gatinel, "Objective assessment of crystalline lens opacity level by measuring ocular light scattering with a double-pass system," American Journal of Ophthalmology, vol. 155 , no. 4 , pp. $629-635,2013$.

[32] J. Fernández, M. Rodríguez-Vallejo, J. Martínez, A. Tauste, J. García-Montesinos, and D. P. Piñero, "Agreement and repeatability of objective systems for assessment of the tear film," Graefe's Archive for Clinical and Experimental Ophthalmology, vol. 256, no. 8, pp. 1535-1541, 2018.

[33] A. Benito, G. M. Pérez, S. Mirabet et al., "Objective optical assessment of tear-film quality dynamics in normal and mildly symptomatic dry eyes," Journal of Cataract \& Refractive Surgery, vol. 37, no. 8, pp. 1481-1487, 2011.

[34] N. Goñi, A. Bidaguren, B. Macías-Murelaga, T. Alberdi, I. Martinez-Soroa, and J. Mendicute, "Objective optical quality analysis using double-pass technique in pterygium surgery," Cornea, vol. 34, no. 1, pp. 60-64, 2015.

[35] J. H. Cho, S. H. Bae, H. K. Kim, and Y. J. Shin, "Optical quality assessment in patients with macular diseases using optical quality analysis system," Journal of Clinical Medicine, vol. 8, no. 6, p. 892, 2019.

[36] S. Koh, N. Maeda, T. Kuroda et al., "Effect of tear film breakup on higher-order aberrations measured with wavefront sensor," American Journal of Ophthalmology, vol. 134, no. 1, pp. 115-117, 2002.

[37] J. S. Yoon, H. Lew, and S. Y. Lee, "Bell's phenomenon protects the tear film and ocular surface after frontalis suspension surgery for congenital ptosis," Journal of Pediatric Ophthalmology \& Strabismus, vol. 45, no. 6, pp. 350-355, 2008. 